\title{
Pion-Baryon Couplings
}

\author{
Alfons J. Buchmann ${ }^{a *}$ and Ernest M. Henley ${ }^{b \dagger}$ \\ ${ }^{a}$ Institute for Theoretical Physics, University of Tübingen, \\ D-72076 Tübingen, Germany \\ and \\ ${ }^{b}$ Department of Physics and Institute for Nuclear Theory, \\ University of Washington, Box 351560, Seattle, WA 98195, USA
}

\begin{abstract}
We have extended and applied a general QCD parameterization method to the emission of pions from baryons. We use it to calculate the strength and sign of the coupling of pions to the octet and decuplet of baryons. Certain relations between octet and decuplet couplings are pointed out.
\end{abstract}

*email:alfons.buchmann@uni-tuebingen.de

†email:HENLEY@nucthy.phys.washington.edu 
In 1989 Morpurgo [1,2] introduced a parameterization for the properties of hadrons, which expresses masses, magnetic moments, transition amplitudes, and other properties of the baryon octet and decuplet in terms of a few parameters. The method uses only general features of QCD and baryon descriptions in terms of quarks. Recently, Dillon and Morpurgo have shown that the method is independent of the choice of the quark mass renormalization point in the QCD Lagrangian [2]. They have also extended the method to nucleon electromagnetic form factors and radii [3].

In addition to the electromagnetic properties of baryons, i.e., the interaction with an external photon field, it is possible to consider the interaction of a baryon with an external pion field, and to calculate the pion-baryon couplings. Due to the internal quark structure of the pion, this problem is rather different from the ones treated in Refs. [1] [3]. Despite the additional difficulties due to the pion's size and mass, the Morpurgo method is nevertheless applicable here, as we argue below.

The Morpurgo method is based on the following considerations. For the observable at hand one formally writes a QCD operator $\Omega$ and QCD eigenstates expressed explicitly in terms of quarks and gluons. This matrix element can, with the help of the unitary operator $V$, be reduced to a calculation in the basis of auxiliary (model) three-quark states $\Phi_{B}$

$$
\langle B|\Omega| B\rangle=\left\langle\Phi_{B}\left|V^{\dagger} \Omega V\right| \Phi_{B}\right\rangle=\left\langle W_{B}|\mathcal{O}| W_{B}\right\rangle .
$$

Both the unitary operator $V$ and the model states $\Phi_{B}$ are defined in Ref. [1]. The $\Phi_{B}$ are pure $L=0$ three-quark states excluding any quark-antiquark or gluon components. $W_{B}$ stands for the standard three-quark $S U(6)$ spin-flavor wave functions. The operator $V$ dresses the auxiliary states $\Phi_{B}$ with $q \bar{q}$ components and gluons and thereby generates the exact QCD eigenstate. Furthermore, the operator $V$ contains a Foldy-Wouthuysen transformation, which transforms the original 4-component Dirac spinor $B$ into a two-component Pauli spinor contained in $W_{B}$.

One then writes the most general expression for $\mathcal{O}$ compatible with the space-time and inner QCD symmetries. The orbital and color space matrix elements are absorbed in unknown parameters multiplying the various invariants appearing in the expansion of $\mathcal{O}$. As an example, for the squared charge radius $\mathcal{O}$ we need a scalar operator linear in the quark charge $Q_{i}$ [3]. The lowest order one is just $\sum_{i} Q_{i}$. The next higher order expression is $\sum_{i \neq j} Q_{i} \boldsymbol{\sigma}_{i} \cdot \boldsymbol{\sigma}_{j}$, where the sum is over all quarks. A three-body term is $\sum_{i \neq j \neq k} Q_{i} \boldsymbol{\sigma}_{j} \cdot \boldsymbol{\sigma}_{k}$, so that the full expression reads

$$
r_{B}^{2}=A \sum_{i} Q_{i}+B \sum_{i \neq k} Q_{i} \boldsymbol{\sigma}_{i} \cdot \boldsymbol{\sigma}_{k}+C \sum_{i \neq j \neq k} Q_{i} \boldsymbol{\sigma}_{j} \cdot \boldsymbol{\sigma}_{k}+\cdots .
$$

The expectation value of the three-quark term is expected to be $\leq 1 / 3$ of the two-quark term, which in turn should be $\leq 1 / 3$ of the one-body term. The reasons for this hierachy of expressions are discussed in Ref. [2].

Coming back to the nucleon pion coupling and writing the standard effective pseudovector coupling

$$
H=-\frac{f}{m_{\pi}} \bar{\psi} \gamma_{\mu} \gamma_{5} \psi \partial^{\mu} \vec{\phi} \cdot \vec{\tau}
$$


it appears that, for a nucleon at rest and in the limit of small four-momentum transfer to the pion, the operator $\Omega$-no matter how complicated- must be such that

$$
\langle N|\Omega| N\rangle=\frac{f}{m_{\pi}} \boldsymbol{\sigma} \cdot \nabla \vec{\tau} \cdot \vec{\phi}
$$

Eq.(3) defines the coupling constant $f$ of the (point) pion field $\vec{\phi}$ to the nucleon, $\boldsymbol{\sigma}$ is the nucleon spin and $\vec{\tau}$ the isospin matrix. It is understood that the right-hand side of Eq.(3) is calculated between the spin-isospin state of the nucleon. In the limit of small four-momentum transfer $\boldsymbol{\nabla} \cdot \vec{\phi}=i \mathbf{k} \cdot \vec{\phi}$. As noted in Ref. [1], even if the right-hand side is non-covariant, referring to the rest frame, the theory is relativistically complete. There can be no other spin-structure for the pion-nucleon interaction, in the limit of small $\mathbf{k}$.

Because the right-hand side of Eq.(3) has a structure similar to the magnetic moment operator, we proceed as in Refs. [1,3]. As shown in detail by Morpurgo [1], the most general parameterization of the axial vector coupling operator $\mathcal{O}$ for octet and decuplet baryons Eq.(3) can be classified in terms of one-quark, two-quark, and three-quark terms plus Trace (closed loop) terms. The latter are not present here.

For the case under discussion the one-body operator can be taken as

$$
\mathcal{O}_{1}=A_{1} \sum_{i} \vec{\tau}^{i} \boldsymbol{\sigma}^{i} \cdot \mathbf{k}
$$

where the sum is over the 3 quarks present in the auxiliary state $\Phi_{B}$. For our purpose, we can use

$$
\mathcal{O}_{1}=A_{1} \sum_{i} \tau_{3}^{i} \sigma_{z}^{i} k_{z}
$$

With this $\mathcal{O}_{1}$, the most general two-body term is given by

$$
\mathcal{O}_{2}=A_{2} \sum_{i, j \neq i} \tau_{3}^{i} \sigma_{z}^{j} k_{z}
$$

Although one can make up further two-body terms, e.g., $\left(\overrightarrow{\tau^{i}} \times \overrightarrow{\tau^{j}}\right)_{3}\left(\boldsymbol{\sigma}^{i} \times \boldsymbol{\sigma}^{j}\right)_{z} k_{z}$, Dillon and Morpurgo [3] have shown that they are not independent. One can also make up three-body terms as $\mathcal{O}_{3}=A_{3} \tau_{3}^{i} \sigma_{z}^{i} k_{z} \boldsymbol{\sigma}^{j} \cdot \boldsymbol{\sigma}^{k}$ and others; we shall neglect them here. In that case, the operator we need is

$$
\mathcal{O}=\mathcal{O}_{1}+\mathcal{O}_{2}
$$

The auxiliary (model) states $\Phi_{B}$ used for calculating the expectation values of the operator $\mathcal{O}$ coincide with the familar $S U(6)$ states multiplied by a spatial wave function with orbital angular momentum $L=0$. The $S U(6)$ states are listed, e.g., in Ref. [1]. We only need the completely symmetric spin-isospin part, $W_{B}$. The radial and color parts of the matrix elements are absorbed in the constants $A_{1}$ and $A_{2}$.

We present, separately, the quark model matrix elements of the operator in Eq.([) to first order (one-body terms) and second order corrections (two-body terms) in Table I. $A_{1}$ and $A_{2}$ are constants to be determined below, and $r$ is the ratio $\frac{m_{u}+m_{d}}{2 m_{s}}=\frac{m_{u}}{m_{s}}$, where $m_{i}$ is the mass of quark i. The reason we include $r$ in the two-body term is that a two-body gluon 
exchange between particles 1 and 2 would be inversely proportional to the masses of the two quarks. This approximate way to take into account $\mathrm{SU}(3)$ symmetry breaking works quite well for magnetic moments [5]. We neglect SU(3) symmetry breaking in the one-body term. A more rigorous treatment of SU(3) symmetry breaking requires the introduction of two constants [1].

In order to obtain from the quark model matrix elements in Table I the conventional pionbaryon couplings, additional overall factors are needed. The pion-octet baryon couplings are defined for spin projection $m_{s}=+1 / 2$ and maximal isospin projection of the corresponding baryon level operator evaluated between baryon spin and isospin wave functions. Similarly, the $\Delta \Delta \pi$ coupling is defined for maximal spin and isospin projection [6].

If we neglect the two-body operators, then the pion coupling to the nucleon is sufficient to fix the unknown constant $A_{1}$ of the theory. When two-body corrections are included, we use the decay of the $\Delta(1232)$ to fix the second constant $A_{2}$. The $N \Delta \pi$ coupling is of the form

$$
H_{N \Delta \pi}=-\frac{f_{N \Delta \pi}}{m_{\pi}} \mathbf{S}^{\dagger} \cdot \nabla \overrightarrow{\mathbf{T}}^{\dagger} \cdot \vec{\phi}+\text { h.c. }
$$

where $\mathbf{S}^{\dagger}$ and $\overrightarrow{\mathbf{T}}^{\dagger}$ are transition spin and isospin operators; they are defined such that their matrix elements are simple Clebsch-Gordan coefficients [7]. The coupling $f_{N \Delta \pi}$ is taken to be $2 \mathrm{f}$, which gives the $\Delta(1232)$ its experimental width of about $130 \mathrm{MeV}$ [7].

Without two-body terms, $A_{1}$ is fixed by $\frac{f^{2}}{4 \pi}=0.08$, and $A_{1}=(3 / 5) f$. The first entry in Table I is equal to $g_{A}$, the axial coupling constant in the additive quark model. In this approximation, one obtains the well known additive quark model result for the $N \Delta \pi$ coupling $f_{N \Delta \pi}^{2}=(72 / 25) f^{2}$. If we include the two-body terms, then we also need the empirical relation $f_{N \Delta \pi} \approx 2 f$ to fix $A_{2}$. In this case, we obtain $A_{1} \approx 0.53 f$ and $A_{2} \approx-0.18 f$, so that $A_{2} / A_{1} \approx 1 / 3$, a quite substantial correction to the additive quark model. For exact $\mathrm{SU}(3)$ symmetry, $r=1$, but if $\mathrm{SU}(3)$ is broken, then $r \approx 330 / 550 \approx 0.6$.

Table II lists the various couplings in terms of $f$, the $\pi^{0} p$ coupling constant, to first order and to second order with and without the inclusion of $r$. We recall that for the decuplet-octet transition couplings, baryon level spin and isospin Clebsch-Gordan coefficients $\left(10 S S_{z} \mid S^{\prime} S_{z}^{\prime}\right)\left(10 T T_{z} \mid T^{\prime} T_{z}^{\prime}\right)$ are needed in order to convert the quark level matrix elements in Table I to the (baryon level) coupling constants listed in Table II. Here, $S(T)$ refers to octet, and $S^{\prime}\left(T^{\prime}\right)$ to decuplet baryons. Similarly, in order to obtain the baryon level decupletdecuplet couplings one uses a conversion factor $1 /\left(T_{z}^{\prime} S_{z}^{\prime}\right)$ [6]. For example, the entry for $\Delta^{+}$ in Table II contains a factor $4 / 3$ to go from quarks to the $\Delta^{+}$. Without $\mathrm{SU}(3)$ breaking the decuplet-decuplet $(D D)$ couplings can then be generically written as $f_{D D}=4 / 3(A+2 B)$, which implies, e.g., $f_{\Delta^{++} \Delta^{++} \pi^{0}}=f_{\Delta^{+} \Delta^{+} \pi^{0}}$.

Our results satisfy the following relation in the $\mathrm{SU}(3)$ symmetric case

$$
f_{\pi^{0} p}+f_{\pi^{0} \Xi^{0}}=f_{\pi^{0} \Sigma^{+}} .
$$

Note that the second-order correction is especially large for the $\Xi^{0}$, because the coefficient in front of $A_{2}$ is 4 times as large as that in front of $A_{1}$. The two-quark operator $\mathcal{O}_{2}$ changes the $\pi^{0} \Delta^{+} \Delta^{+}$coupling from the first order value $f_{\pi \Delta \Delta}=(4 / 5) f$ to the total result $f_{\pi \Delta \Delta}=0.23 f$. We agree with Brown and Weise [6] for those pion couplings they calculated using a onequark operator, namely to the nucleon and $\Delta$. 
The $\pi \Sigma \Lambda$ and the $\pi \Sigma^{*} \Lambda$ couplings remain unaffected by $\mathrm{SU}(3)$ symmetry breaking. Irrespective of the value of $r$ our octet-decuplet transition couplings satisfy the sum rule

$$
\sqrt{2} f_{\Delta^{+} p}=f_{\Xi^{* 0} \Xi^{0}}+\sqrt{6} f_{\Sigma^{*+} \Sigma^{+}}-f_{\Sigma^{* 0} \Lambda^{0}} .
$$

This relation is not new. It has been derived before [8] using $\mathrm{SU}(3)$ symmetry and its breaking to first order.

Next, we compare our results for the transition couplings to those obtained in the large $N_{c}$ approach [9]. Because the couplings in [9] are computed for $\pi^{+}$emission, we calculate the matrix elements in Eq.(11) with $\tau_{z}$ in Eq.(可) replaced by $\tau_{+}$. We then obtain for the transition $\Delta^{++} \rightarrow p \pi^{+}: 4 \sqrt{3}(A-B) / 3$, for $\Sigma^{*+} \rightarrow \Sigma^{0} \pi^{+}:-2 \sqrt{2}(A-(2 r-1) B) / 3$, and finally for $\Sigma^{*+} \rightarrow \Lambda^{0} \pi^{+}:-2 \sqrt{6}(A-B) / 3$. By taking ratios of two transition couplings we get for the case $r=1$

$$
\frac{\Delta^{++} \rightarrow p}{\Sigma^{*+} \rightarrow \Sigma^{0}}=-\sqrt{6} \quad(-3.06), \quad \frac{\Sigma^{*+} \rightarrow \Sigma^{0}}{\Sigma^{*+} \rightarrow \Lambda^{0}}=-\frac{1}{\sqrt{3}} \quad(-0.46)
$$

The numbers in parentheses include $\mathrm{SU}(3)$ symmetry breaking in the two-quark term $(r=$ 0.6). These results are in agreement with those obtained in the large $N_{c}$ approach [9], including the next-to-leading order corrections, which is undoubtedly more than a numerical coincidence.

In Table III we compare the couplings we obtain with the inclusion of the two-body terms to those derived by other means. The values of the coupling constants from Stoks and Rijken (SR) [10] are obtained from fits to baryon-nucleon scattering data and one-boson exchange potentials. The difference in sign from SR in the entry to Table III is because they use the coupling for $\pi^{+} \Sigma^{+} \Lambda^{0}$. The SR couplings are essentially the same as those of Maessen, Rijken, and de Swart [11]. The columns labeled KDOL [12 are obtained from QCD sum rules with the use of $\mathrm{SU}(3)$ and "beyond" $\mathrm{SU}(3)$ by correcting for mass effects. Our values tend to be closer to those of Stoks and Rijken [10]. The latter also satisfy Eq.(91) in contrast to the $\mathrm{SU}(3)$ symmetric values of Ref. [12].

Finally, we point out certain analytical relations between octet and decuplet baryon couplings to pions that emerge from our theory (neglecting three-quark terms)

$$
\begin{aligned}
f_{\pi^{0} p}-\frac{1}{4} f_{\pi^{0} \Delta^{+} \Delta^{+}} & =\frac{\sqrt{2}}{3} f_{\pi^{0} p \Delta^{+}} \\
f_{\pi^{0} \Sigma^{+}}-\frac{1}{2} f_{\pi^{0} \Sigma^{*+} \Sigma^{*+}} & =\frac{1}{\sqrt{6}} f_{\pi^{0} \Sigma^{*+} \Sigma^{+}} \\
f_{\pi^{0} \Xi^{0}}-\frac{1}{4} f_{\pi^{0} \Xi^{* 0} \Xi^{* 0}} & =\frac{1}{3} f_{\pi^{0} \Xi^{* 0} \Xi^{0} .} .
\end{aligned}
$$

They are a consequence of the underlying unitary symmetry, and are valid for all values of the strange quark mass. Eq.(12) can be used to predict the elusive decuplet couplings from the experimentally better known octet and decuplet-octet transition couplings. As far as we know, these relations are new.

In summary, we have used the Morpurgo formalism to predict pion-baryon coupling constants. The inclusion of two-body operators leads to significant corrections of the additive quark model values. Finally, we hope that this work will stimulate further research along 
these lines, such as the inclusion of three-quark operators and a more rigorous treatment of $\mathrm{SU}(3)$ flavor breaking.

Acknowledgement: This work has been partially supported by a U.S. DOE grant. We would like to thank Drs. G. Morpurgo and G. Dillon for useful criticism and valuable suggestions. 


\section{REFERENCES}

[1] G. Morpurgo, Phys. Rev. D40, 2997 (1989); Phys. Rev. D40, 3111 (1989).

[2] G. Morpurgo, Phys. Rev. Lett. 68, 139 (1992); Phys. Rev. D46, 4068 (1992); G. Dillon and G. Morpurgo, Phys. Rev. D53, 3754 (1996).

[3] G. Dillon and G. Morpurgo, Phys. Lett. B448, 107 (1999); Phys. Lett. B459, 321 (1999); Z. Phys. C73, 547 (1997).

[4] D. B. Lichtenberg, Unitary Symmetry and Elementary Particles, Academic Press, New York, 1978; F. E. Close, An introduction to Quarks and Partons, Academic Press, London, 1979.

[5] G. Wagner, A. Buchmann, and A. Faessler, Phys. Lett. B359, 288 (1995).

[6] G. E. Brown and W. Weise, Phys. Rep. C22, 279 (1975).

[7] T. E. O. Ericson and W. Weise, Pions and Nuclei, Clarendon Press, Oxford, 1988.

[8] V. Gupta and V. Singh, Phys. Rev. 135 (1964) B1442; C. Becchi, E. Eberle, and G. Morpurgo, Phys. Rev. 136 (1964) B808.

[9] R. Dashen, E. Jenkins, and A. V. Manohar, Phys. Rev. D49 (1994) 4713.

[10] V. G. J. Stoks and Th. A. Rijken, Phys. Rev. C59, 3009 (1999).

[11] P.M.M. Maessen, Th.A. Rijken, and J.J. de Swart, Phys. Rev. C40, 2226 (1989).

[12] H. Kim, T. Doi, M. Oka, and S.H. Lee, LANL Arch. nucl-th/9909007 


\section{TABLES}

\begin{tabular}{|l|r|c|}
\hline \hline Baryon & First order & Second order \\
\hline $\mathrm{p}$ & $\frac{5}{3} A_{1}$ & $-\frac{2}{3} A_{2}$ \\
$\Sigma^{+}$ & $\frac{4}{3} A_{1}$ & $\frac{2(2-r)}{3} A_{2}$ \\
$\Sigma^{0} \rightarrow \Lambda^{0}$ & $-\frac{2 \sqrt{3}}{3} A_{1}$ & $\frac{2 \sqrt{3}}{3} A_{2}$ \\
$\Xi^{0}$ & $-\frac{1}{3} A_{1}$ & $\frac{4 r}{3} A_{2}$ \\
\hline$\Delta^{+} \rightarrow p$ & $\frac{4 \sqrt{2}}{3} A_{1}$ & $-\frac{4 \sqrt{2}}{3} A_{2}$ \\
$\Sigma^{*+} \rightarrow \Sigma^{+}$ & $\frac{2 \sqrt{2}}{3} A_{1}$ & $\frac{2 \sqrt{2}(1-2 r)}{3} A_{2}$ \\
$\Sigma^{* 0} \rightarrow \Lambda^{0}$ & $\frac{2 \sqrt{6}}{3} A_{1}$ & $-\frac{2 \sqrt{6}}{3} A_{2}$ \\
$\Xi^{* 0} \rightarrow \Xi^{0}$ & $\frac{2 \sqrt{2}}{3} A_{1}$ & $-\frac{2 \sqrt{2} r}{3} A_{2}$ \\
\hline$\Delta^{+}$ & $A_{1}$ & $2 A_{2}$ \\
$\Sigma^{*+}$ & $2 A_{1}$ & $2(1+r) A_{2}$ \\
$\Xi^{* 0}$ & $A_{1}$ & $2 r A_{2}$ \\
\hline \hline
\end{tabular}

TABLE I. Table of quark model matrix elements of the operator in Eq.(đ) to first order $\left(\mathcal{O}_{1}\right)$ and second order corrections $\left(\mathcal{O}_{2}\right)$.

\begin{tabular}{|l|r|r|r|}
\hline \hline Baryon & $\begin{array}{r}\text { First order } \\
\left(A_{2}=0\right)\end{array}$ & Total & Total \\
& $\mathrm{r}=1$ & $\mathrm{r}=0.6$ \\
\hline $\mathrm{p}$ & 0.80 & 1 & 1 \\
$\Sigma^{+}$ & -0.69 & 0.59 & 0.54 \\
$\Sigma^{0} \rightarrow \Lambda^{0}$ & -0.20 & -0.82 & -0.82 \\
$\Xi^{0}$ & 1.70 & -0.42 & -0.32 \\
\hline$\Delta^{+} \rightarrow p$ & 0.98 & $2^{*}$ & $2^{*}$ \\
$\Sigma^{*+} \rightarrow \Sigma^{+}$ & -1.20 & 1.16 & 0.92 \\
$\Sigma^{* 0} \rightarrow \Lambda^{0}$ & -1.20 & -1.42 & -1.42 \\
$\Xi^{* 0} \rightarrow \Xi^{0}$ & 0.80 & -1.42 & -1.28 \\
\hline$\Delta^{+}$ & 0.80 & 0.23 & 0.23 \\
$\Sigma^{*+}$ & 0.80 & 0.23 & 0.32 \\
$\Xi^{* 0}$ & 0.23 & 0.42 \\
\hline \hline
\end{tabular}

TABLE II. Coupling constants of the pion to various members of the octet and decuplet, and the decuplet-octet transitions in terms of $f=f_{\pi^{0} p}$. The ${ }^{*}$ indicates an input. The ratio $r=m_{u} / m_{s}$ of non-strange and strange quark masses indicates the degree of flavor symmetry breaking. 


\begin{tabular}{|l|l|l|l|l|}
\hline \hline & SR [10] & \multicolumn{2}{|c|}{ KDOL [12] } & this work \\
\hline & & SU(3) & broken SU(3) & \\
\hline$\Sigma^{+}$ & 0.71 & 0.28 & 0.83 & 0.54 \\
$\Sigma^{0} \rightarrow \Lambda^{0}$ & -0.75 & - & - & -0.82 \\
$\Xi^{0}$ & -0.29 & -0.46 & -2.05 & -0.32 \\
\hline \hline
\end{tabular}

TABLE III. Coupling constants of the pion to baryons in terms of $f_{\pi^{0} p}$ as found by various authors. 